\title{
Particle Morphology of Spray-Freeze Dried Microencapsulation Agents
}

\author{
Hilal Isleroglu (Corresponding author) \\ Tokat Gaziosmanpasa University, Faculty of Natural Sciences and Engineering, \\ Food Engineering Dept., 60150, Tokat, Turkey \\ E-mail: hilal.isleroglu@gop.edu.tr \\ Izzet Turker \\ Tokat Gaziosmanpasa University, Faculty of Natural Sciences and Engineering, \\ Food Engineering Dept., 60150, Tokat, Turkey \\ E-mail: izzet.turker2615@gop.edu.tr
}

\begin{abstract}
The research is financed by Tokat Gaziosmanpasa University Scientific Research Projects (Project Number: 2016/62) and the Scientific and Technological Research Council of Turkey (TUBITAK, Project Number: 115O216).
\end{abstract}

\begin{abstract}
Spray-freeze drying (SFD) is a relatively new technique for microencapsulation of heat sensitive products. In SFD process, the solution is sprayed in a cryogenic medium such as liquid nitrogen to form frozen fine droplets. Then, the droplets subjected to freeze-drying under vacuum. The freeze-drying time is comparatively short due to the relatively large surface area achieved by the SFD. In this study, frequently used microencapsulation agents namely maltodextrin, inulin and gum arabic were sprayfreeze dried both individual and in the form of mixtures and the resulting powders were characterized with respect to particle morphologies. Morphology of the particles were observed using Scanning Electron Microscopy (SEM). All the spray-freeze dried particles showed spherical shape, sometimes being slightly aggregated. Regular pore structures originating from the formation of crystals were observed on the surface of the maltodextrin particles, while the gum arabic particles were found to have more irregular and small pores. However, inulin particles were found to have a smoother surface form than others.
\end{abstract}

Keywords: Spray-freeze drying, morphology, inulin, maltodextrin, gum Arabic

DOI: $10.7176 / \mathrm{JSTR} / 5-2-24$

\section{Introduction}

The spray-freeze drying process (SFD) is a relatively new drying method that combines the properties of conventional freeze-drying and spray drying processes, resulting in the formation of micro or nano-scale particles in a spherical and porous form (Anandharamakrishnan, 2008). The SFD process basically takes place in three stages. The solution prepared with the material to be dried is sprayed by a nozzle to form fine droplets. The resulting droplets are frozen by means of a cooling fluid (cryogenic medium). The sprayed droplets are then dried under reduced pressure to give the product in powder form (Wanning et al., 2015). Small droplets obtained by spraying of the solution provide more homogenous and excess surface area during the freezing phase and increase both freezing and subsequent drying rates. The formation of small sized spheres results in an increase in surface mass transfer coefficient, resulting in a reduction in total drying time compared to conventional freeze-drying. Drying of small sized spheres obtained by spraying process can produce uniform and free flowing powders (MacLeod et al., 2006; Her et al., 2010). Furthermore, the spherical and porous structures of the particles obtained by the SFD process increase the rehydration capacity of the powder product. The SFD process is generally used to 
the pharmaceutical industry, but it has recently been introduced in the food industry (Ishiwarya et al., 2015). In addition to the products used in the pharmaceutical industry such as soluble drugs, inhaled drugs and insulin; some biomaterials used in the food industry such as soluble coffee, whey proteins, egg albumin, vitamins, bovine serum albumin, trypsinogen, lysozyme and microorganisms were also successfully dried by the SFD process (Sonner et al., 2002; Yu et al., 2006; Engstrom et al., 2007; Semyonov et al., 2010; D’Addio et al., 2012; Parthasarathi \& Anandharamakrishnan, 2016; Teixeira et al., 2017).

The morphology of the particles obtained by the SFD process depends on the methods applied during the spraying, freezing and drying processes. Furthermore, the composition of the feed solution and the dry matter ratio affect the surface morphology of the particle. It was determined that the porosity of the particles obtained by the SFD process increased as the dry matter content of the feed solution decreased (Mueannoom et al., 2012). Freezing rate significantly affects the droplet morphology. Nucleation is a process that is highly dependent on temperature and occurs randomly. In case of a significant temperature gradient within the droplet due to rapid cooling, the initial nucleation starts on the surface of the droplet. If the temperature distribution is constant, homogeneous nucleation may occur in the interior of the droplet. Surface nucleation causes the formation of characteristic patterns in the pores of the dried particles (Ishiwarya et al. 2015). If the freezing stage is carried out properly, there is no change in the microstructure during the freeze-drying phase except the removal of water (Anandharamakrishnan et al., 2010). In a study, the morphology of whey powder obtained by conventional spray drying and SFD process is compared using scanning electron microscopy (SEM). The SEM images revealed a smooth, skin forming behavior of spray-dried powder with slight deflation due to the collapse of the shell during loss of moisture from the particle. In the samples dried by the SFD process, a porous microstructure and a surface layer surrounding this porous microstructure were detected (Al-Hakim \& Stapley, 2004). Different materials such as lysozyme (D'Addio et al., 2012), whey protein isolate (Anandharamakrishnan, 2008), whole fat milk (Rogers et al., 2008) and polyethylene glycol (Barron et al., 2003) were dried by SFD resulting in particles having a uniform spherical structure and a large number of pores on the surface. It was stated that these porous structures shorten the drying time compared to the conventional freeze-drying process (Anandharamakrishnan, 2008).

Although the use of SFD for microencapsulation is a relatively new method, it is one of the most successful methods in terms of microencapsulation efficiency and protection of the core material (Ishwarya \& Anandharamakrishnan, 2015). The coating agents used in microencapsulation processes affect the functional, physical and sensory properties of the end product. Thus, the microencapsulation agents; (i) should maintain their rheological properties even at high concentrations, (ii) should have high emulsification ability and form stable emulsions with the core material, (iii) should not react with the core material during the microencapsulation process and at subsequent stages (storage, etc.), (iv) should provide protection against stress factors by forming a tight structure in drying processes, (v) should be cheap, inert and easy to find (Desai \& Park, 2005). Carbohydrates increase the adhesion between the microencapsulation agents and the core material, as well as providing a spherical and smooth microcapsule formation (Bruschi et al., 2003). In the microencapsulation process, carbohydrates such as starch, maltodextrin and corn syrup are generally preferred. These materials are used as microencapsulation agents due to their low viscosity and good solubility even at high concentrations (Balasubramani et al., 2015; Mahdavi et al., 2016; Kalušević et al., 2017). Also, gums having high viscosity are generally used as microencapsulation agents. The gum arabic, also known as the acacia gum, is frequently preferred because of its high emulsifying power (Dickinson, 2003). Although the individual coating material has many of the desirable properties, no single material can supply all the properties required of an ideal encapsulating agent (Desai \& Park, 2005). Therefore, approaches to improving encapsulating properties have focused on blends of these materials for microencapsulation.

In this study, maltodextrin, inulin and gum arabic, which are frequently used as microencapsulation agents, were dried by SFD method as an individual or in the form of mixtures. It was aimed to determine how the SFD process, which is quite new in food applications, affects the morphology of these materials. The particle morphologies obtained as a result of SFD process were determined which would affect the final product quality if they were used as a mixture in the microencapsulation studies. 


\section{Materials and Methods}

\subsection{Materials}

Commercial powder forms of maltodextrin (DE: 20), inulin and gum arabic were used as microencapsulation agents and the solutions having $10 \%$ dry matter of these agents were prepared for SFD process. The individual solutions were agitated for 5 minutes at $11200 \mathrm{rpm}$ using rotor-stator homogenizer (Ultra-Turrax IKA T-18 Basic, USA). Also, binary $(50: 50 \mathrm{w} / \mathrm{w})$ and triple $(33: 33: 33 \mathrm{w} / \mathrm{w})$ mixtures were prepared by homogenizing $(5 \mathrm{~min}, 11200 \mathrm{rpm})$ the whole mixture $(10 \%$ dry matter $)$ after adding the each component to the mixture in equal ratio.

\subsection{Spray-Freeze Drying}

The prepared solutions were fed with a syringe pump (Syringe Pump TI, SonoTek Inc., USA) at a constant flow rate of $6.83 \mathrm{ml} / \mathrm{min}$ to an ultrasonic nozzle working at $48 \mathrm{kHz}$ (SonoTek Inc., USA). The feed solution was atomized into the vapor above the boiling liquid nitrogen $\left(\sim-196^{\circ} \mathrm{C}\right)$ which was located in a stainless steel container approximately $500 \mathrm{ml}$ in amount. The fine droplets began rapidly freezing in the vapor headspace region above the liquid nitrogen, and the freezing finished completely when immersed in the liquid. The liquid nitrogen was agitated gently to prevent collapse of the frozen droplets. After freezing, the excess liquid nitrogen was removed by evaporation from the slurry containing the frozen droplets, and the resulting pellet was transferred to a pre-cooled shelf $\left(\sim-35^{\circ} \mathrm{C}\right)$ of a freeze dryer (CHRIST, Alpha 1-4 LSC, Germany). The frozen particles were subjected to main drying for 12 hours at 1 mbar and then to secondary drying for 4 hours, with a shelf temperature at $30^{\circ} \mathrm{C}$ under a vacuum of 0.01 mbar. The schematic diagram of the SFD system used in this study was given by Isleroglu et al. (2018)

\subsection{Determination of particle morphology}

The particle morphology of the spray-freeze dried powders was determined using a scanning electron microscopy (SEM) (Zeiss Evo LS 10, Germany). Samples were placed on to aluminum stages using adhesive carbon conducting tape. Particles were coated with gold for 200 seconds and the images were taken at reduced pressure of $9.75 \times 10^{-5}$ torr and at an accelerating voltage of $20 \mathrm{kV}$.

\section{Results and Discussion}

One of the benefits of SFD technique is the ability to produce particles having different microstructures than those produced by other conventional techniques such as spray drying and freeze-drying. The most important advantage of SFD is the formation of pores on the outer surface of the particles in powder products, which makes this technique a more specific scientific value than others (Dutta et al., 2018). This is attributed to the gaps left behind during the sublimation (drying step) of the ice crystals resulting from exposure to the cold medium (Anandharamakrishnan et al., 2010). These pores then facilitate the rehydration of the product, allowing for rapid and effective dissolution (Anandharamakrishnan, 2008). To demonstrate the difference between the particle morphologies of conventional freeze-drying and SFD processes, maltodextrin solution was frozen at $-80^{\circ} \mathrm{C}$ for 4 hours without spraying, and freeze-dried under the same conditions as SFD. The final product was broken up into particles by mechanical crushing after drying process. The light microscope images of both freeze-dried and SFD maltodextrin are shown in Fig 1. While the maltodextrin particles obtained by SFD were in spherical form, it was observed that those obtained with conventional freeze-drying were in flakey structure. Although large-size crystal structures were observed in the conventional freeze-dried maltodextrins, the crystals obtained by SFD were not large enough to be detected by light microscopy (Fig 1). MacLeod et al. (2006) stated that the fine droplets resultant from spraying offer more homogeneous temperature field for heat transfer during the spray-freezing step which leads to uniform nucleation and formation of fine ice crystals.

To propound the morphological properties of the samples obtained by SFD, the SEM images were achieved. As a result of the SFD process, it has been determined that particles can be obtained in spherical form having uniform distribution (Fig 2 and Fig 3). The surface morphology of the maltodextrin obtained by SFD was determined in our previous study (Türker et al., 2018). While the pores on the surface of maltodextrin and gum arabic (Fig 3) particles were observed, the surface of inulin (Fig 2) particles was

201 | P a g e

www.iiste.org 
found to be smoother. When the SEM images obtained for maltodextrin were examined, it was seen that a very regular pore structure was formed on the surface (Türker et al., 2018). The SEM image given in Türker et al. (2018) clearly showed that the fast freezing rates during SFD achieved much smaller pore sizes as a result of small crystals than that achieved from conventional freeze-drying (Fig 1). However, the formation of the crystal structure is a stress factor for sensitive materials such as enzymes and may have negative effects on the activity (Tonnis et al., 2015). When the SEM images obtained from the microencapsulation agents were examined for inulin, it was seen that smooth particles which were spherical, but did not contain pores unlike gum arabic and maltodextrin (Fig 2c). Since the inulin is a flexible oligosaccharide, the glassy structure has resulted in a more compact surface matrix and less pore particles. In addition, a certain degree of agglomeration was observed by the interaction of the inulin molecules with each other (Fig 2a). This was the result of the high bonding capacity of inulin molecules. Lacerda et al. (2016), in which they performed microencapsulation of jussara pulp with different coating materials, supported the formation of more intense agglomeration with SEM images by increasing the inulin concentration. Additionally, the most important parameters affecting the particle size are the nozzle frequency, feed rate, viscosity and surface tension of the feed solution (Masters, 1991). Hence, larger particle size was obtained by gum arabic which has higher viscosity than maltodextrin and inulin (Fig 3). The determination of the microstructure of the products produced by SFD provides information on whether or not the structural precipitation occurred during the freeze-drying process, and also allows the flow and solubility properties of the powder product to be determined visually (Ishwarya et al., 2015). When different types of carbohydrates are used as microencapsulation agents for protein-containing solutions, they affect the protein stability in a different way (Desai \& Park, 2005). According to the glass transition theory, when proteins form matrix with carbohydrates, the molecular movement of the proteins is reduced and the degradation rates decrease. It is thought that the amorphous structure, which is formed when using inulin as a microencapsulation agent, protects the protein-based molecules from mechanical stresses such as ice and dehydration stress during drying. However, the rigid polysaccharides such as maltodextrin may interact with proteins, but leave some open regions (Tonnis et al., 2015). There are studies showing the success of inulin in protecting proteins especially in freeze-drying processes (Furlán et al., 2012; Grasmeijer et al., 2013; Tonnis et al., 2014). The SEM images of the binary and triple combinations of maltodextrin, gum arabic and inulin are shown in Fig 4. When inulin and gum arabic were used in a binary combination, a smoother surface was obtained than the others (Fig 4a). When the three components were used in a combination, larger and irregular pores were observed on the surface (Fig 4d). According to the desired properties in the microencapsulated product, these coating materials can be used in a binary or triple combination. For example, if the material to be coated is a compound having a biological activity, the pores on the surface may not be preferred for the stability. In this case, only inulin or a mixture of gum arabic and inulin may be used. However, if it is desired to improve the physical and reconstitution properties, mixtures which provide more porosity on the surface should be preferred.

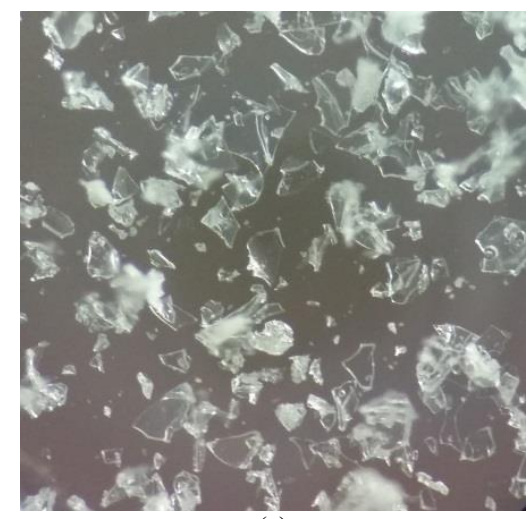

(a)

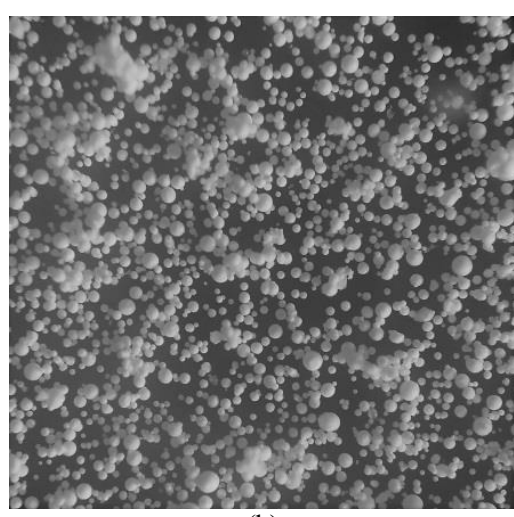

(b)

Figure 1. Light microscope images of maltodextrin (100x) a) conventional freeze-drying b) sprayfreeze drying 


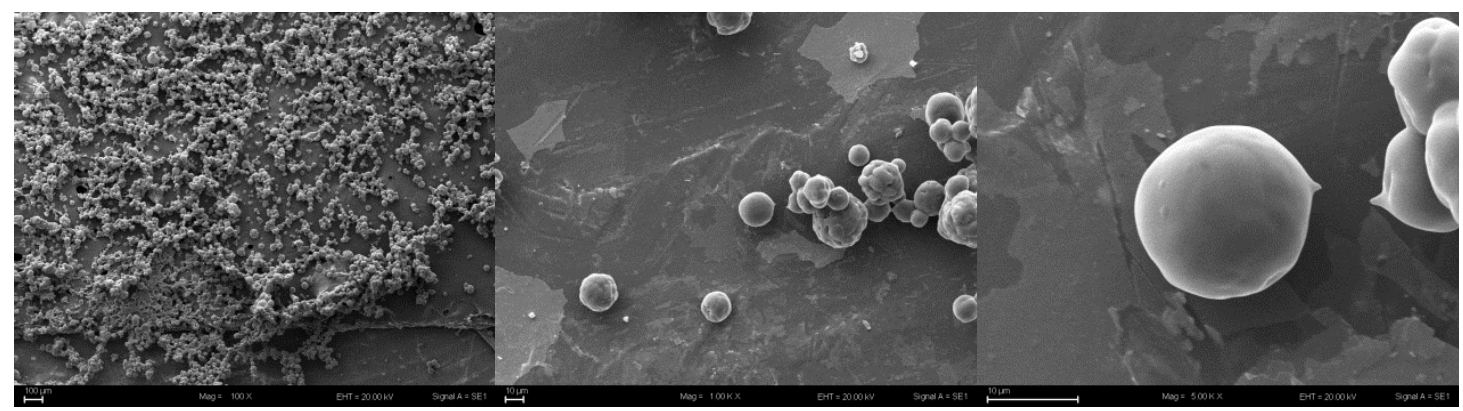

(a)

(b)

(c)

Figure 2. SEM images of inulin obtained by SFD (a) 100x, (b) 1000x, c) 5000x magnification

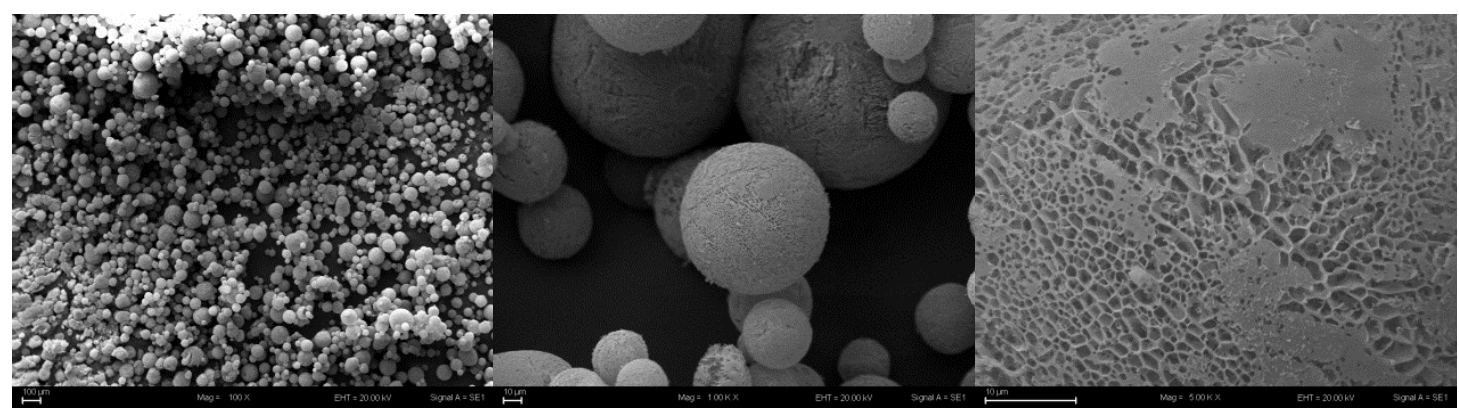

(a)

(b)

(c)

Figure 3. SEM images of gum arabic obtained by SFD (a) 100x, (b) 1000x, c) 5000x magnification
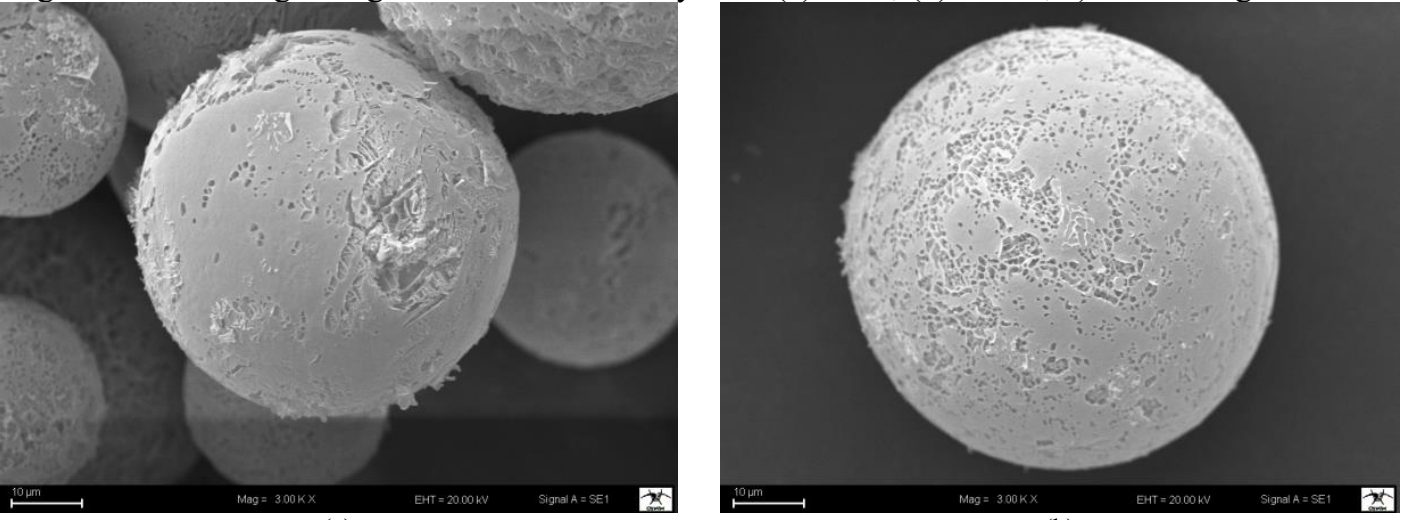

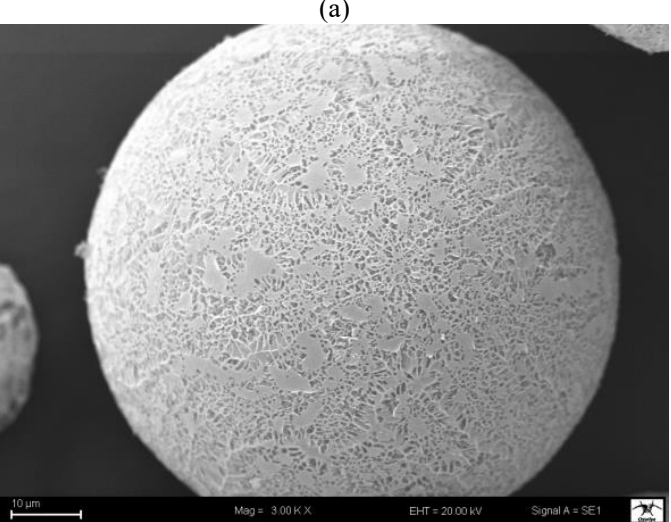

(c)

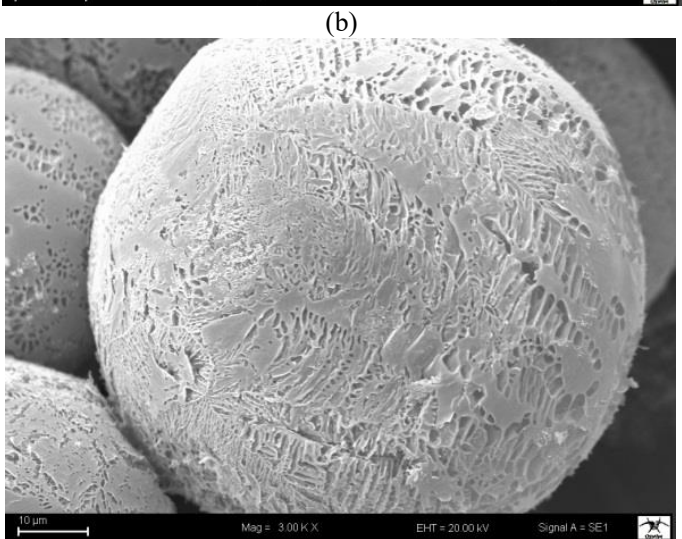

(d)

Figure 4. SEM images of the combinations of microencapsulation agents (3000x magnification) (a) gum arabic+inulin, (b) gum arabic+maltodextrin, c) maltodextrin+inulin, d) maltodextrin+gum arabic+inulin

203 | P a g e

www.iiste.org 


\section{Conclusion}

In this study, maltodextrin, inulin and gum arabic, which are frequently used as microencapsulation agents, were dried by SFD method and the particle morphologies were examined. As a result, all the samples were observed to be in a spherical shape. It was observed that gum arabic particles were larger in size due to higher viscosity of gum arabic, and inulin molecules were smaller and more prone to aggregation. Although different forms of porous structures were observed on the surfaces of maltodextrin and gum arabic particles, inulin particles were found to be smoother. The surface porosity aids in solubilization for rehydration during end use. However, it may not be desirable in the case of protection of a biological activity. Therefore, the microencapsulation agent or mixture to be used should be selected according to the desired final product property.

\section{References}

Al-Hakim K. \& Stapley, A.G.F. (2004). Morphology of spray-dried and spray-freeze-dried whey powders. Drying 2004-Proceedings of the 14th international drying symposium, 1720-1726, August.

Anandharamakrishnan C., Rielly, C.D. \& Stapley A.G.F. (2010). Spray-freeze-drying of whey proteins at sub-atmospheric pressures. Dairy Science and Technology, 90(2-3), 321-334.

Anandharamakrishnan, C. (2008). Experimental and computational fluid dynamics studies on sprayfreeze-drying and spray-drying of whey proteins. Doctoral dissertation, Loughborough University, Leichester, UK.

Balasubramani, P., Palaniswamy, P.T., Visvanathan, R., Thirupathi, V., Subbarayan, A. \& Maran, J.P. (2015). Microencapsulation of garlic oleoresin using maltodextrin as wallmaterial by spray drying technology. International Journal of Biological Macromolecules, 72, 210- 217.

Barron, M.K., Young, T.J., Johnston, K.P. \& Williams, R.O. (2003). Investigation of processing parameters of spray freezing into liquid to prepare polyethylene glycol polymeric particles for drug delivery. AAPS PharmSciTech, 4(2), 1-13.

Bruschi, M.L., Cardoso, M.L.C., Lucchesi, M.B. \& Gremiao, M.P.D. (2003). Gelatin microparticles containing propolis obtained by spray-drying technique: preparation and characterization. International Journal of Pharmaceutics, 264, 45-55.

D’Addio, M.S., Chan, Y.G.J., Kwok, L.C.P., Prud'homme, K.R. \& Chan, H.K. (2012). Constant size, variable density aerosol particles by ultrasonic spray freeze drying. International Journal of Pharmaceutics, 427(2), 185- 191.

Desai, K.G.H. \& Park, H.J. (2005). Recent developments in microencapsulation of food ingredients, Drying Technology, 23, 1361-1394.

Dickinson, E. (2003). Hydrocolloids at interfaces and the influence on the properties of dispersed systems. Food Hydrocolloids, 17, 25-39.

Dutta, S., Moses, J.A. \& Anandharamakrishnan, C. (2018). Modern frontiers and applications of spray-freeze-drying in design of food and biological supplements. Journal of Food Process Engineering, e12881.

Engstrom, J.D., Simpson, D.T., Lai, E.S., Williams, R.O. \& Johnston, K.P. (2007). Morphology of protein particles produced by spray freezing of concentrated solutions. European Journal of Pharmaceutics and Biopharmaceutics, 65(2), 149-162. 
Furlán, L.T.R., Lecot, J., Padilla, A.P., Campderrós, M.E. \& Zaritzky, N. (2012).Stabilizing effect of saccharides on bovine plasma protein: A calorimetric study. Meat Science, 91 (4), 478-485.

Grasmeijer, N., Stankovic, M., de Waard, H., Frijlink, H.W. \& Hinrichs, W.L. (2013). Unraveling protein stabilization mechanisms: Vitrification and water replacement in a glass transition temperature controlled system. BBA-Proteins and Proteomics, 1834 (4), 763-769.

Her, J.Y., Song, C.S., Lee, S.J. \& Lee, K.G. (2010). Preparation of kanamycin powder by an optimized spray freeze-drying method. Powder Technology. 199(2), 159-164.

Ishiwarya, S.P., Anandharamakrishnan, C. \& Stapley, A.G.F. (2015). Spray-freeze drying - A novel process for the drying of foods and bioproducts. Trends in Food Science and Technology, 41(2), $161-181$

Isleroglu, H., Turker, I., Tokatli, M. \& Koc, B. (2018). Ultrasonic spray-freeze drying of partially purified microbial transglutaminase. Food and Bioproducts Processing, 111, 153-164.

Kalušević, A.M., Lević, S.M., Čalija, B.R., Milić, J.R., Pavlović, V.B., Bugarski, B.M. \& Nedović, V.A., 2017. Effects of different carrier materials on physicochemical properties of microencapsulated grape skin extract. Journal Food Science and Technology, 54 (11), 3411-3420.

Lacerda, E.C.Q., de Araújo Calado, V.M., Monteiro, M., Finotelli, P.V., Torres, A.G. \& Perrone, D. (2016). Starch, inulin and maltodextrin as encapsulating agents affect the quality and stability of jussara pulp microparticles. Carbohydrate Polymers, 151, 500-510.

MacLeod, C., McKittrick, J., Hindmarsh, J., Johns, M. \& Wilson, D. (2006). Fundamentals of spray freezing of instant coffee. Journal of Food Engineering, 74 (4), 451-461.

Mahdavi, S.A., Jafari, S.M., Assadpoor, E. \& Dehnad, D. (2016). Microencapsulation optimization of natural anthocyanins with maltodextrin, gum arabic and gelatin. International Journal of Biological Macromolecules, 85, 379-385.

Masters, K. (1991). Spray drying handbook (5th ed.). Harlow: Longman Scientific \& Technical, 725p, New York, USA.

Mueannoom, W., Srisongphan, A., Taylor, K.M., Hauschild, S. \& Gaisford S. (2012). Thermal inkjet spray freeze-drying for preparation of excipient-free salbutamol sulphate for inhalation. European Journal of Pharmaceutics and Biopharmaceutics, 80(1), 149-155.

Parthasarathi, S. \& Anandharamakrishnan, C. (2016). Enhancement of oral bioavailability of vitamin E by spray-freeze drying of whey protein microcapsules. Food and Bioproducts Processing, 100, 469-476.

Rogers, S., Wu, W.D., Saunders, J. \& Chen, X.D. (2008). Characteristics of milk powders produced by spray freeze drying. Drying Technology, 26(4), 404-412.

Semyonov, D., Ramon, O., Kaplun, Z., Levin-Brener, L., Gurevich, N. \& Shimoni, E. (2010). Microencapsulation of Lactobacillus paracasei by spray freeze drying. Food Research International, 43(1), 193-202.

Sonner, C., Maa, Y.F. \& Lee, G. (2002). Spray-freeze-drying for protein powder preparation: Particle characterization and a case study with trypsinogen stability. Journal of Pharmaceutical Sciences, 91(10), 2122-2139. 
Teixeira, C.C.C., de Freitas Cabral, T.P., Tacon, L.A., Villardi, I.L., Lanchote, A.D. \& de Freitas, L.A.P. (2017). Solid state stability of polyphenols from a plant extract after fluid bed atmosphericspray-freeze-drying. Powder Technology, 319, 494-504.

Tonnis, W.F., Amorij, J.P., Vreeman, M.A., Frijlink, H.W., Kersten, G.F. \& Hinrichs, W.L.J. (2014). Improved storage stability and immunogenicity of hepatitis B vaccine after spray-freeze drying in presence of sugars. European Journal of Pharmaceutical Sciences, 55, 36-45.

Tonnis, W.F., Mensink, M.A., de Jager, A., van der Voort Maarschalk, K., Frijlink, H. W. \& Hinrichs, W.L.J. (2015). Size and molecular flexibility of sugars determine the storage stability of freezedried proteins. Molecular Pharmaceutics, 12 (3), 684-694.

Türker, İ., Koç, B., \& İşleroğlu, H. (2018). Effect of spray freeze drying process on physical properties of maltodextrin. Gida / The Journal of Food, 197-210.

Wanning, S., Lamprecht, A. \& Süverkrüp R. (2015). Pharmaceutical spray freeze drying. International Journal of Pharmaceutics, 488, 136-153.

Yu, Z., Johnston, K.P. \& Williams RO. (2006). Spray freezing into liquid versus spray-freeze drying: influence of atomization on protein aggregation and biological activity. European Journal of Pharmaceutical Sciences, 27(1), 9-18. 